\title{
Behavioral Dietary Intervention
}

National Cancer Institute

\section{Source}

National Cancer Institute. Behavioral Dietary Intervention. NCI Thesaurus. Code C67021.

The use of operant conditioning to alter a person's dietary choices and behaviors. 